\title{
Caractérisation du système enzymatique protéolytique d'Aeromonas hydrophila LP 50
}

\author{
par \\ Françoise Denis et Louise VeIllet-Poncet*
}

\section{INTRODUCTION}

Dans une précédente publication nous avons étudié la nature et l'évolution du système enzymatique protéolytique d'Aeromonas hydrophila LP 50, en fonction du développement de la culture dans le temps.

A. hydrophila LP 50, cultivé sur milieu à la polypeptone glucosé, produit un système enzymatique protéolytique exocellulaire complexe, doué d'activités endopeptidasique et aminopeptidasique, mais dépourvu d'activité carboxypeptidasique.

Ce mémoire définit les caractéristiques enzymatiques de ce système protéolytique.

\section{MATERIEL ET METHODES}

\section{Matériel d'étude. Cultures. Extraits enzymatiques}

La souche retenue, Aeromonas hydrophila LP 50, isolée de laits pasteurisés conditionnés [16], est cultivée dans les conditions décrites précédemment : culture en fioles Erlenmeyer de 21 , contenant 11 de milieu à la polypeptone glucosé et maintenues à $30^{\circ} \mathrm{C}$ dans un bainmarie thermostaté, sans agitation.

L'obtention des extraits enzymatiques bruts, utilisés pour déterminer les activités protéolytiques, se fait dans les mêmes conditions que précédemment : précipitation des protéines des filtrats acellulaires par addition de sulfate d'ammonium, dialyse contre du tampon véronal $0,05 \mathrm{M} \mathrm{pH} 8$ à $5^{\circ} \mathrm{C}$.

* Laboratoire de Microbiologie Alimentaire, E.N.S.A.I.A. - I.N.P.L., Nancy. 


\section{Caractérisation du système protéolytique}

Nous étudions les activités protéolytiques en fonction des paramètres suivants : la température, le $\mathrm{pH}$ et l'action de substances capables d'agir spécifiquement sur des résidus d'acides aminés faisant partie intégrante du site actif.

Les conditions de dosage des différentes activités sont celles mentionnées antérieurement : dosage de l'activité endopeptidasique sur caséine Hammarsten (composés solubles dans les filtrats trichloracétiques à 10 p. 100 estimés avec le réactif de Folin, selon la méthode de Lowry [10]), sur hémoglobine dénaturée, selon une méthode dérivée de celle d'Anson [1] (teneur en peptides de la fraction soluble dans les filtrats trichloracétiques à 5 p. 100 mesurée à $280 \mathrm{~nm}$ ), dosage de l'activité aminopeptidasique sur L-leucine-paranitroanilide (variation de densité optique mesurée à $410 \mathrm{~nm}$ [11]).

La méthode de dosage de l'activité endopeptidasique sur hémoglobine ne nous donnant pas entière satisfaction, nous réalisons les dosages d'activité endopeptidasique sur un troisième substrat, l'azocaséine (Serva) par une modification de la méthode de Charney et Tomarelly [2]. Un ml d'une solution d'azocaséine à 1 p. 100 en tampon tris- $\mathrm{HCl} 0,2 \mathrm{M}$ pH 8 est ajouté à $1 \mathrm{ml}$ d'extrait enzymatique brut convenablement dilué dans le tampon véronal $0,05 \mathrm{M} \mathrm{pH} 8$. Après un temps d'action de 20 min à différentes températures, entre 20 et $65^{\circ} \mathrm{C}$, la réaction est arrêtée par addition de 2,5 $\mathrm{ml}$ d'acide trichloracétique à $5 \mathrm{p} .100$. Après filtration du mélange sur papier Whatman $\mathrm{n}^{\circ} 3,1,5 \mathrm{ml}$ de filtrat est ajouté à $1,5 \mathrm{ml}$ de $\mathrm{NaOH} 0,5 \mathrm{~N}$. La teneur en produits solubles du filtrat trichloracétique est mesurée par la variation d'absorbance à $440 \mathrm{~nm}$.

L'unité d'activité endopeptidasique est arbitrairement définie comme la variation de 0,01 unité de densité optique dans les conditions décrites ci-dessus.

\subsection{INFLUENCE DE LA TEMPÉRATURE}

Suivant les activités protéolytiques préalablement déterminées en fonction de la croissance, nous choisissons quatre extraits enzymatiques : l'extrait obtenu à partir d'une culture de $24 \mathrm{~h}$ (début de la phase stationnaire de la courbe de croissance), traitée par sulfate d'ammonium à 60 p. 100 de saturation $\left(24_{60}\right)$, l'extrait obtenu à partir d'une culture de $36 \mathrm{~h}$ (correspondant au milieu de la phase stationnaire et à l'optimum des activités protéolytiques maxima enregistrées), traitée par sulfate d'ammonium à 50 p. 100 de saturation $\left(36_{50}\right)$, l'extrait obtenu à partir d'une culture de $36 \mathrm{~h}$ pour une zone de précipitation $50-60$ p. 100 de saturation en sulfate d'ammonium $\left(36_{60}\right)$, l'extrait obtenu à partir d'une culture de $54 \mathrm{~h}$ (correspondant à la chute brusque du début de la phase de déclin), traitée par sulfate d'ammonium à 60 p. 100 de saturation $\left(54_{60}\right)$. 
Les essais de stabilité thermique sont réalisés en maintenant les extraits enzymatiques à différentes températures pendant des temps compris entre 5 et $50 \mathrm{~min}$, puis nous mesurons l'activité résiduelle dans les conditions usuelles de dosage, à la ternpérature optima d'activité.

\subsection{INFLUENCE DU PH}

L'influence du pH sur les activités protéolytiques est testée pour des valeurs de $\mathrm{pH}$ comprises entre 5,5 et 12, en utilisant les tampons suivants : tampon citrate-phosphate $0,05 \mathrm{M}$ (pH 5,5-6-6,5-7-7,5), tampon tris- $\mathrm{HCl} 0,05 \mathrm{M}$ (pH 7-7,5-8-8,2-8,4-8,6-8,8-9), tampon glycine- $\mathrm{NaOH} 0,05 \mathrm{M}$ (pH 8,5-9-9,5-10-10,5-11-12).

Les essais de stabilité en fonction du $\mathrm{pH}$ sont réalisés en portant les extraits enzymatiques à différentes valeurs de $\mathrm{pH}$ dans l'intervalle $6-10,5$. Les préparations sont conservées $30 \mathrm{~min}$ à température ambiante et $20 \mathrm{~min}$ à $45^{\circ} \mathrm{C}$, puis amenées au $\mathrm{pH}$ optimum de réaction par addition de soude ou d'acide chlorhydrique $0,2 \mathrm{~N}$. L'activité enzymatique résiduelle est déterminée, dans les conditions usuelles de dosage, à la température et au $\mathrm{pH}$ optimums de réaction.

\subsection{Action des inhibiteurs des enzymes protéolytiques}

L'influence de trois inhibiteurs d'activité protéolytique, l'E.D.T.A. (Serva) (acide éthylène-diamine-tétra-acétique), le P.M.S.F. (Serva) (fluorure de phényl-méthyl-sulfonyl) et la L-cystéine- $\mathrm{HCl}$ (Serva) est déterminée de la façon suivante : $0,5 \mathrm{ml}$ d'extrait enzymatique convenablement dilué avec le tampon tris- $\mathrm{HCl} 0,05 \mathrm{M} \mathrm{pH}=7,5$ est ajouté à $0,5 \mathrm{ml}$ de chaque inhibiteur, aux concentrations de $0,1 \mathrm{mM}, 1 \mathrm{mM}$ et $10 \mathrm{mM}$, préparées en milieu tampon tris- $\mathrm{HCl} 0,05 \mathrm{M} \mathrm{pH} \mathrm{7,5}$ pour l'E.D.T.A. et la L-cystéine-HCl et dans l'éthanol à 95 p. 100 pour le P.M.S.F. Le temps d'action est de $20 \mathrm{~min}$ à $45^{\circ} \mathrm{C}$. Les activités protéolytiques résiduelles sont évaluées selon les méthodes usuelles de dosage sur l'azocaséine d'une part et sur la L-leucine-para-nitroanilide d'autre part.

\section{RESULTATS}

\section{Température}

\subsection{Activité ENDOPEPTIDASIQUE}

\subsubsection{Sur caséine Hammarsten}

Nous mettons en évidence (fig. 1) non seulement l'activité plus importante des extraits $36_{50}$ et $54_{60}$, que nous choisissons dans la poursuite de notre étude, mais également pour chacun des extraits enzymatiques une température optima d'action de $50^{\circ} \mathrm{C}$. 
Nous confirmons ce dernier résultat en déterminant l'activité sur caséine, en fonction du temps, à des températures comprises entre $20^{\circ} \mathrm{C}$ et $65^{\circ} \mathrm{C}$ (fig. 2).

La vitesse d'hydrolyse passe bien par un maximum à $50^{\circ} \mathrm{C}$, pour les deux extraits $36_{50}$ et $54_{60}$.

L'activité mesurée, pour l'extrait $36_{50}$, à $55^{\circ} \mathrm{C}$, représente encore 98 p. 100 de l'activité maxima. A $65^{\circ} \mathrm{C}$, l'activité obtenue correspond à 84 p. 100 de l'activité à $50^{\circ}$ C. A $35^{\circ} \mathrm{C}$, elle représente 59 p. 100 de l'activité maxima.

Nous constatons, dans les résultats de stabilité thermique (fig. 3), que l'extrait enzymatique $36_{50}$ présente 100 p. 100 d'activité après avoir été soumis à des températures inférieures ou égales à $45^{\circ} \mathrm{C}$, pendant $15 \mathrm{~min}$, et qu'ensuite, en fonction du temps et de la température à laquelle cet extrait est soumis, nous observons une perte graduelle d'activité endopeptidasique : 5 p. 100 d'activité sont détruits à $50^{\circ} \mathrm{C}$, $13 \mathrm{p} .100$ à $55^{\circ} \mathrm{C}$ et $32 \mathrm{p} .100$ à $65^{\circ} \mathrm{C}$. La perte d'activité est de $60 \mathrm{p} .100$ après action à $70^{\circ} \mathrm{C}$ pendant $20 \mathrm{~min}$ et de $92 \mathrm{p} .100$ pour $80^{\circ} \mathrm{C}$, l'inactivation est totale à $100^{\circ} \mathrm{C}$.

\subsubsection{Sur hémoglobine dénaturée et sur azocaséine}

La température optima d'action, sur hémoglobine dénaturée et azocaséine, se situe à $40^{\circ} \mathrm{C}$ pour les deux extraits $36_{50}$ et $54_{60}$.

Sur azocaséine (fig. 4), l'activité, mesurée à $35^{\circ} \mathrm{C}$ et $55^{\circ} \mathrm{C}$, représente encore $92 \mathrm{p} .100$ de l'activité maxima pour l'extrait $36_{50}$. A $65^{\circ} \mathrm{C}$, l'activité obtenue correspond à 59 p. 100 de l'activité à $40^{\circ} \mathrm{C}$.

Nous remarquons, pour la courbe d'inactivation thermique de l'extrait enzymatique $36_{50}$, sur azocaséine (fig. 5), que la perte d'activité est déjà de 9 p. 100 après action à $30^{\circ} \mathrm{C}$ pendant $15 \mathrm{~min}, 12 \mathrm{p} .100$ à $50^{\circ} \mathrm{C}, 43 \mathrm{p} .100$ à $65^{\circ} \mathrm{C}$, l'inactivation est totale à $80^{\circ} \mathrm{C}$.

\subsection{ACtiVité aminopeptidasiQue}

\subsubsection{Sur L-leucine-para-nitroanilide}

Nous constatons (fig. 6) que l'extrait enzymatique $36_{50}$ présente la quasi-totalité de l'activité aminopeptidasique du système enzymatique d'A. hydrophila LP 50. C'est pourquoi nous utiliserons cet extrait pour étudier l'influence de la température sur l'hydrolyse de la L-leucine-para-nitroanilide, dans l'intervalle $16-65^{\circ} \mathrm{C}$, en fonction du temps.

Les résultats obtenus (fig. 7) montrent que la vitesse d'hydrolyse passe par un maximum à $55^{\circ} \mathrm{C}$. L'activité mesurée à $60^{\circ} \mathrm{C}$ et $65^{\circ} \mathrm{C}$ représente encore respectivement 85 et 60 p. 100 de l'activité maxima. A $35^{\circ} \mathrm{C}$, elle correspond à $43 \mathrm{p} .100$ de l'activité à $55^{\circ} \mathrm{C}$.

Les essais de stabilité thermique (fig. 8) montrent que l'extrait enzymatique $36_{50}$ est stable aux températures inférieures ou égales à $40^{\circ} \mathrm{C}$ pour un temps d'action de $15 \mathrm{~min}$, à $55^{\circ} \mathrm{C}$ l'activité résiduelle 


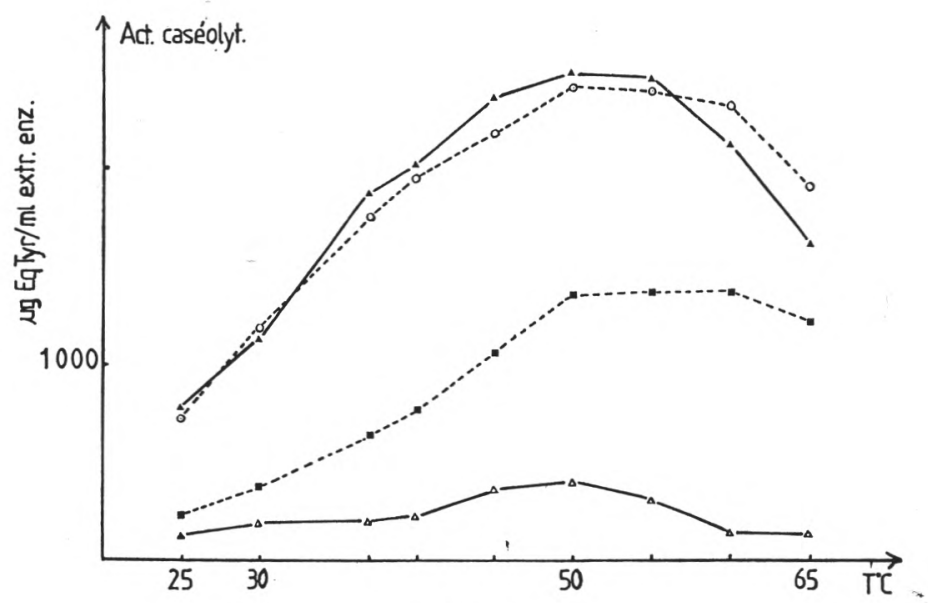

fig. 1

Activité caséolytique des extraits enzymatiques bruts $\mathbf{2} 24_{60}, \boldsymbol{\Delta} 36_{50}$, $\triangle 36_{60}$, $\bigcirc 54_{60}$, en fonction de la température, pour une durée de réaction enzymatique de $10 \mathrm{~min}$.

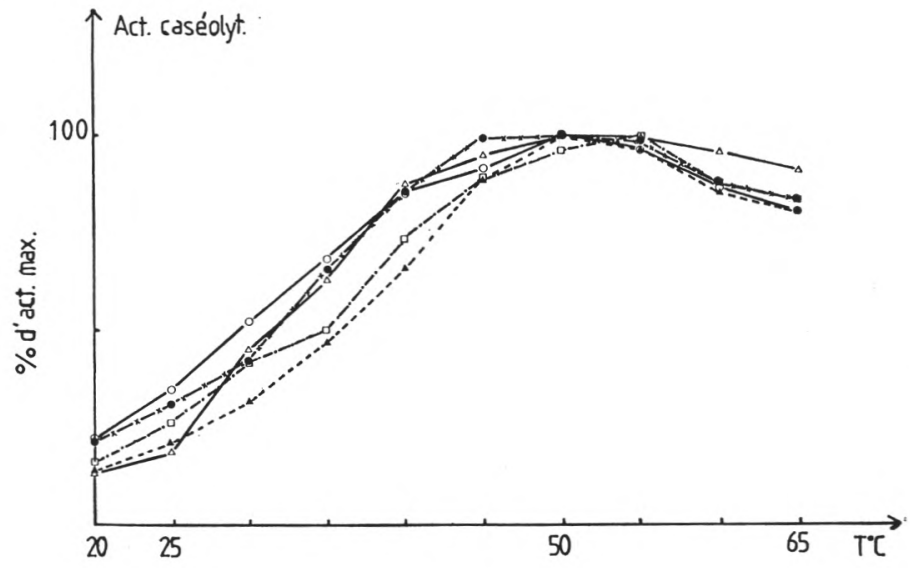

fig. 2

Influence de la température sur l'hydrolyse de la caséine par l'extrait enzymatique brut $36_{50}$ pour différentes durées de réaction enzymatique : $\triangle 5 \mathrm{~min}, \Delta 10 \mathrm{~min}, \quad$ व $15 \mathrm{~min}, \bullet 20 \mathrm{~min}, \quad$ o $25 \mathrm{~min}$. 


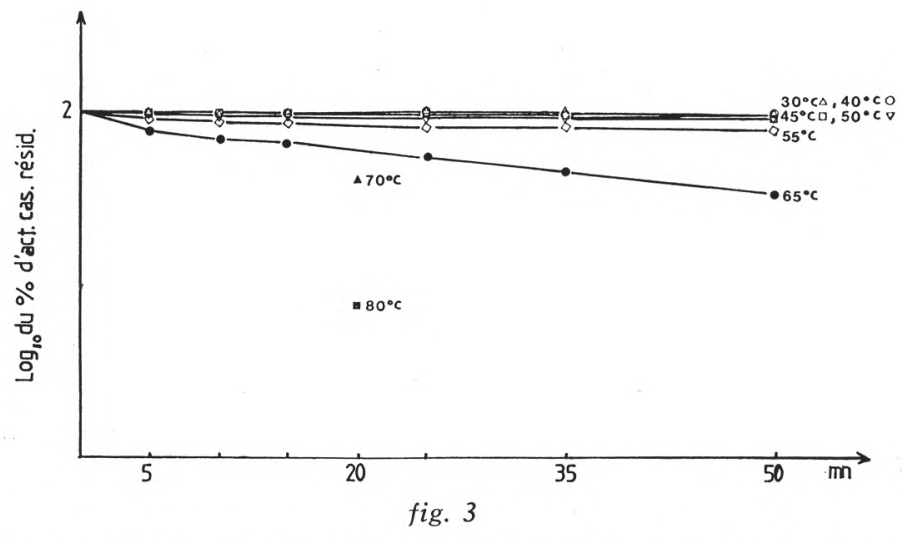

Inactivation thermique de l'extrait enzymatique brut $36_{50}$ en fonction de la durée de chauffage (min), à différentes températures.

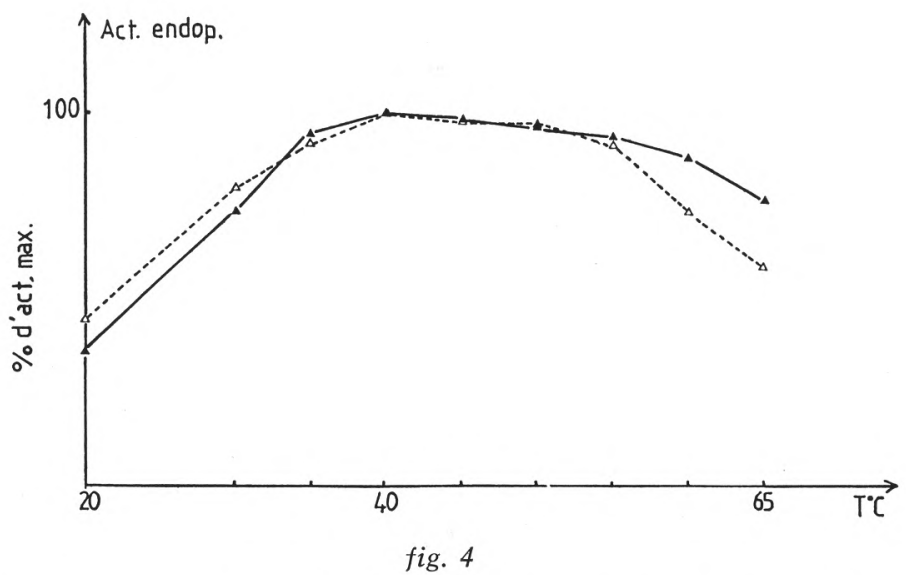

Influence de la température sur l'hydrolyse de l'azocaséine par les extraits enzymatiques bruts $36_{50}(\triangle)$ et $54_{60}(\mathbf{\Delta})$, pour une durée de réaction enzymatique de $20 \mathrm{~min}$. 


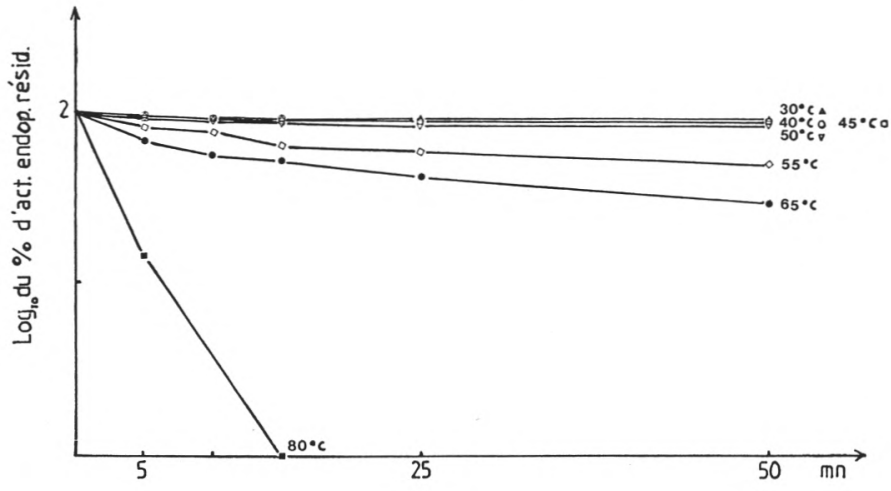

fig. 5

Inactivation thermique de l'extrait enzymatique brut $36_{50}$ en fonction de la durée de chauffage (min), à différentes températures.

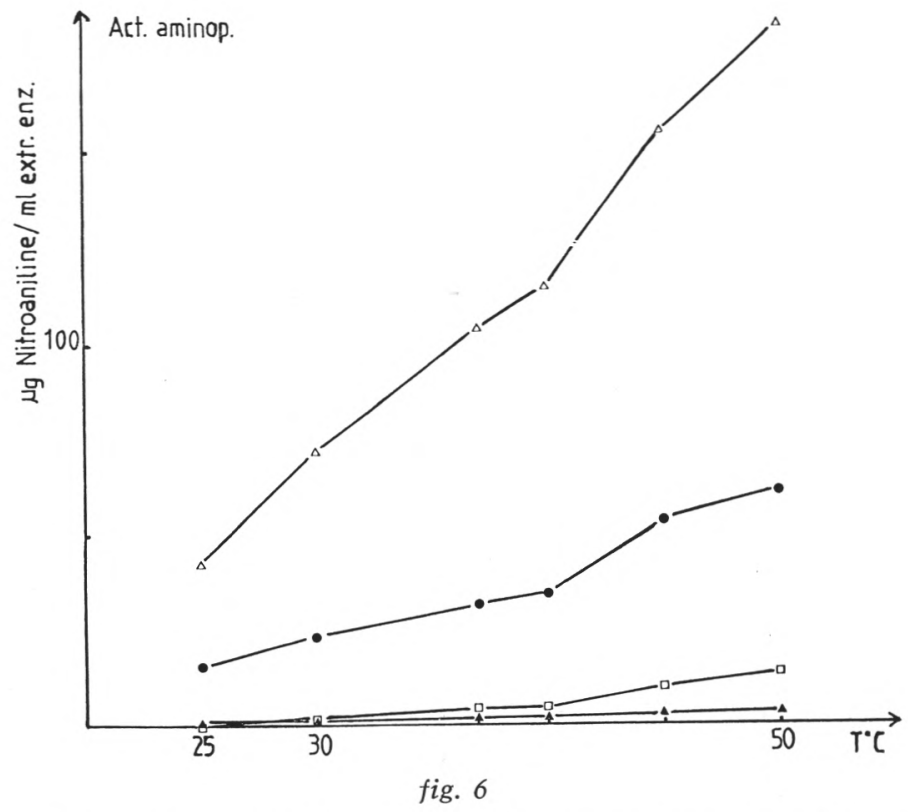

Activité aminopeptidasique des extraits enzymatiques bruts $\square 24_{60}$, $\Delta 36_{50}, \mathbf{\Delta} 36_{60}, \bullet 54_{60}$, en fonction de la température, pour une durée de réaction enzymatique de $15 \mathrm{~min}$. 


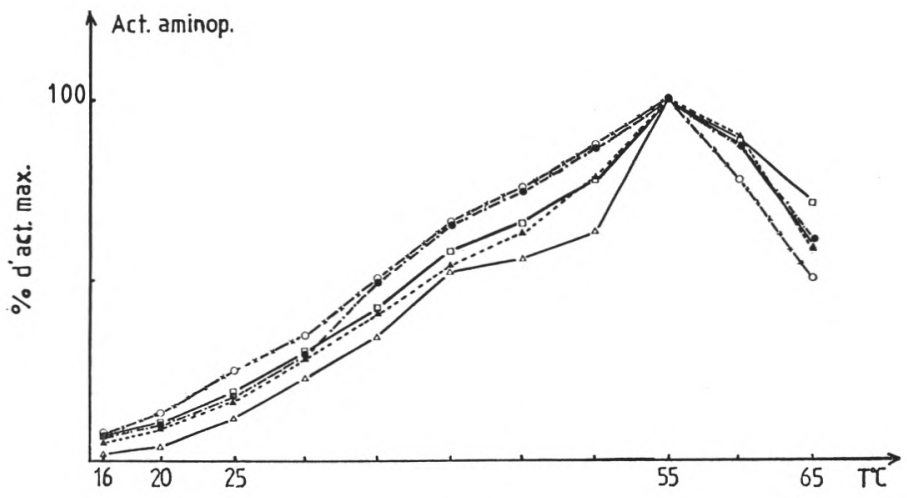

fig. 7

Influence de la température sur l'hydrolyse de la L-leucine-paranitroanilide par l'extrait enzymatique brut $36_{50}$ pour différentes durées de réaction enzymatique : $\triangle 5 \mathrm{~min}, \boldsymbol{\Delta} 10 \mathrm{~min}, \square 15 \mathrm{~min}$, - $20 \mathrm{~min}, \mathrm{O} 25 \mathrm{~min}$.

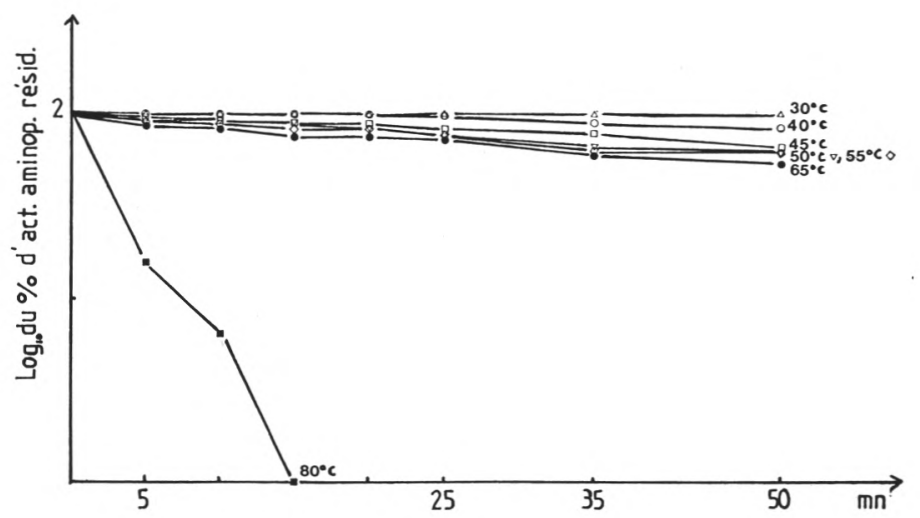

fig. 8

Inactivation thermique de l'extrait enzymatique brut $36_{50}$ en fonction de la durée de chauffage $(\mathrm{min})$ à différentes températures. 
est de 83 p. 100 et de 76 p. 100 à $65^{\circ} \mathrm{C}$. A $80^{\circ} \mathrm{C}$, la destruction thermique de l'activité aminopeptidasique est rapide.

L'activité aminopeptidasique semble moins thermolabile que l'activité endopeptidasique. Ce résultat concorde avec ceux obtenus par Griffin et Prescott [6], Foster et Hanna [4] dans leur étude sur le système enzymatique protéolytique d'A. proteolytica.

\section{2. $\mathrm{pH}$}

\subsection{Activité ENDOPEPTIDASIQue}

\subsubsection{Sur caséine Hammarsten}

Le $\mathrm{pH}$ optimum d'hydrolyse de la caséine, à la température de $50^{\circ} \mathrm{C}$, pour une durée de réaction de $15 \mathrm{~min}$, est voisin de 8 - 8,2 (fig. 9) pour les deux extraits enzymatiques $36_{50}$ et $54_{60}$.

A pH 9, l'activité représente encore 93 p. 100 de l'activité maxima. Aux pH 7,5 et 7, l'activité obtenue correspond respectivement à 84 et 51 p. 100 de l'activité maxima pour l'extrait 36. Aux pH 5,5 (caséine en partie précipitée) et 12 , elle ne représente plus que 18 et 14 p. 100 de l'activité maxima.

La stabilité à différents $\mathrm{pH}$ est maxima dans la zone de $\mathrm{pH} 7$ - 8,5 pour les deux extraits (fig. 10). Dans cet intervalle, l'activité résiduelle est au moins égale à 90 p. 100 de l'activité initiale.

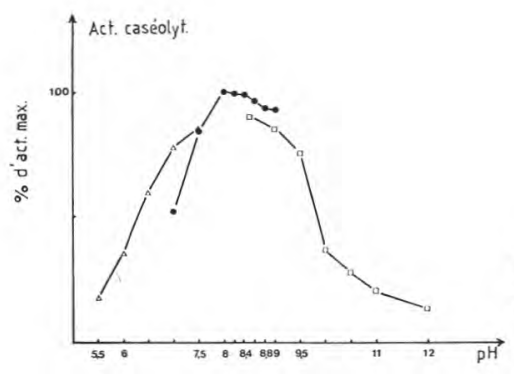

fig. 9

Influence du pH sur l'hydrolyse de la caséine par l'extrait enzymatique brut $36_{50}$. Tampons utilisés : $[0,05 \mathrm{M}] \triangle$ citrate-phosphate, $\bullet$ tris- $\mathrm{HCl}$, 口 glycine- $\mathrm{NaOH}$.

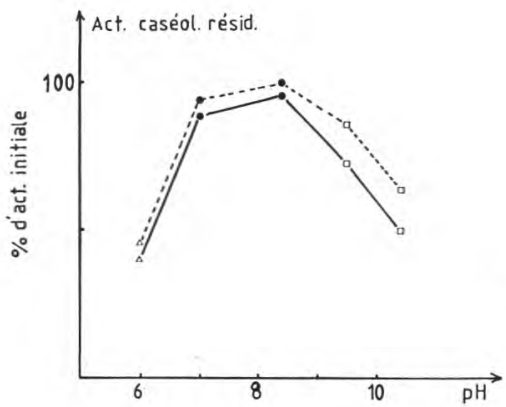

fig. 10

Influence du $\mathrm{pH}$ sur la stabilité des extraits enzymatiques bruts $36_{50}(\longrightarrow$ et $54_{60}(-\ldots)$.

Tampons utilisés : $[0,05 \mathrm{M}] \triangle$ citrate-phosphate, $\bullet$ tris- $\mathrm{HCl}$, 口 glycine- $\mathrm{NaOH}$. 


\subsubsection{Sur azocaséine}

L'hydrolyse de l'azocaséine est effectuée à la température de $40^{\circ} \mathrm{C}$, pour une durée de réaction de $20 \mathrm{~min}$. Nous définissons une zone d'activité maxima située au voisinage de $\mathrm{pH}$ 7,5-8 pour les deux extraits (fig. 11).

Aux pH 7 et 8,2, l'activité mesurée représente encore 97 p. 100 de l'activité maxima pour l'extrait enzymatique $36_{50}$.

A pH 9, elle correspond encore à 73 p. 100 de l'activité maxima. Aux pH 5,5 et 12, l'activité obtenue ne représente plus que 47 et 19 p. 100 de l'activité maxima.

Les deux extraits enzymatiques $36_{50}$ et $54_{60}$ présentent une zone de stabilité maxima s'étendant de $\mathrm{pH} 7$ à $\mathrm{pH} 8,5$ (fig. 12). Dans cet intervalle, l'activité résiduelle atteint 85 à 90 p. 100 de l'activité initiale.

\subsection{Activité AMINOPEPTIDASIQUE}

\subsubsection{Sur L-leucine-para-nitroanilide}

Le $\mathrm{pH}$ optimum d'hydrolyse de la L-LNA, à la température de $55^{\circ} \mathrm{C}$, pour une durée de réaction de $15 \mathrm{~min}$, est de 7,5 pour l'extrait enzymatique $36_{50}$ (fig. 13).

A pH 9, l'activité obtenue représente encore 85 p. 100 de l'activité maxima. Aux pH 7 et 12, elle est respectivement égale à 66 et 13 p. 100 de l'activité maxima. A pH 6 et au-dessous, l'activité aminopeptidasique est nulle.

Dans la zone des pH basiques, 8,5 - 12, l'activité aminopeptidasique est fortement affectée par le tampon utilisé, le tampon borate- $\mathrm{NaOH}$ 0,05 M étant plus favorable que les tampons carbonate-bicarbonate $0,05 \mathrm{M}$ et glycine- $\mathrm{NaOH} 0,05 \mathrm{M}$.

L'extrait enzymatique $36_{50}$ est stable pour les $\mathrm{pH}$ voisins de 7,3 7,5 (fig. 14).

\section{Action des inhibiteurs des enzymes protéolytiques}

Nous notons (fig. 15), pour les deux extraits enzymatiques $36_{50}$ et 5460, que l'E.D.T.A. et le P.M.S.F. présentent un pouvoir inhibiteur, dès la concentration de $0,1 \mathrm{mM}$, sur l'activité endopeptidasique évaluée sur azocaséine.

La L-cystéine- $\mathrm{HCl}$ est sans effet aux concentrations de 0,1 et $1 \mathrm{mM}$; en revanche, à $10 \mathrm{mM}$, l'inactivation est presque totale.

Nous constatons (fig. 16), pour l'activité aminopeptidasique de l'extrait 36, évaluée sur L-LNA, une perte d'activité de 76 p. 100 après action du P.M.S.F. à la concentration de $0,1 \mathrm{mM}$ et une inactivation de l'ordre de 59 p. 100 par l'E.D.T.A. et de 50 p. 100 par la L-cystéine$\mathrm{HCl}$ pour une concentration de $10 \mathrm{mM}$. 

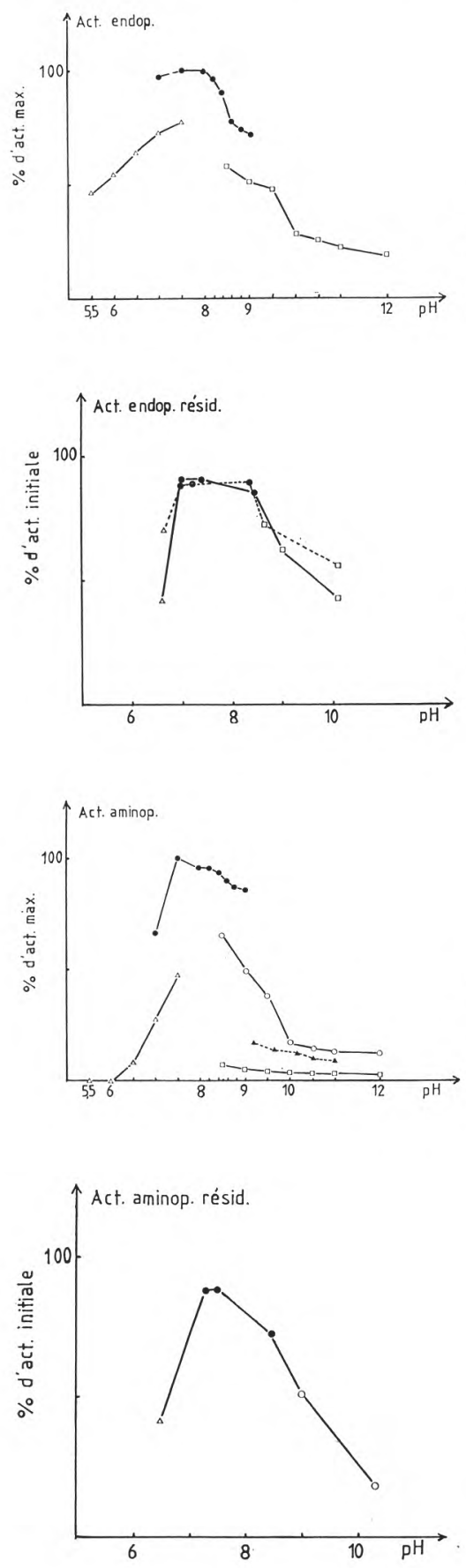

fig. 11

Influence du $\mathrm{pH}$ sur l'hydrolyse de l'azocaséine par l'extrait enzymatique brut $36_{50}$. Tampons utilisés : $[0,05 \mathrm{M}] \triangle$ citrate-phosphate, $\bullet$ tris- $\mathrm{HCl}$, 口 glycine- $\mathrm{NaOH}$.

\section{fig. 12}

Influence du pH sur la stabilité des extraits enzymatiques bruts $36_{50}(-)$ et $54_{60}(---)$.

Tampons utilisés : $[0,05 \mathrm{M}] \triangle$ citrate-phosphate, $\bullet$ tris- $\mathrm{HCl}$, 口 glycine- $\mathrm{NaOH}$.

\section{fig. 13}

Influence du $\mathrm{pH}$ sur l'hydrolyse de la Lleucine-para-nitroanilide par l'extrait enzymatique brut $36_{50}$.

Tampons utilisés : $[0,05 \mathrm{M}] \triangle$ citrate-phosphate, $\bullet$ tris- $\mathrm{HCl}, \mathrm{O}$ borate- $\mathrm{NaOH}, \boldsymbol{\Delta}$ carbonate-bicarbonate, $\square$ glycine- $\mathrm{NaOH}$.

fig. 14

Influence du pH sur la stabilité de l'extrait enzymatique brut $36_{50}$.

Tampons utilisés : $[0,05 \mathrm{M}] \Delta$ citrate-phosphate, tris- $\mathrm{HCl}$, O borate- $\mathrm{NaOH}$. 


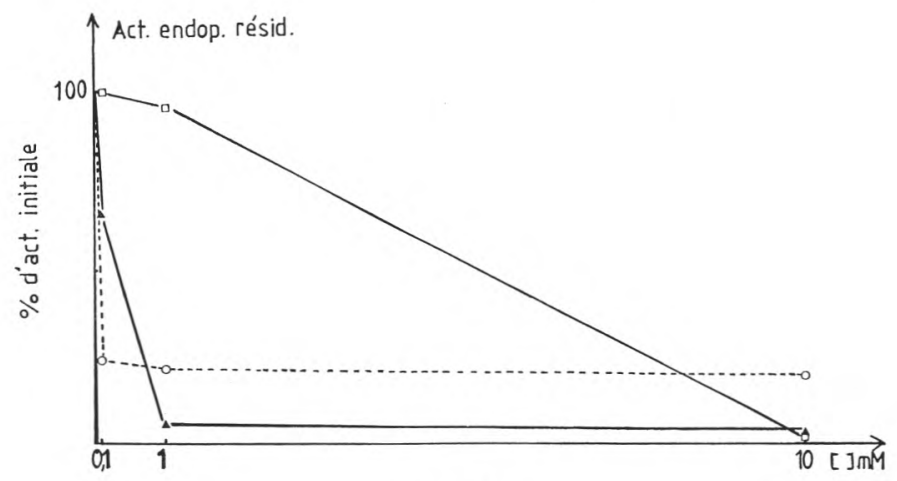

fig. 15

Inactivation de l'extrait enzymatique brut $36_{50}$ par l'E.D.T.A. $\mathbf{\Delta}$ le P.M.S.F. O, la L-cystéine-HCl $\square$ (substrat : azocaséine).

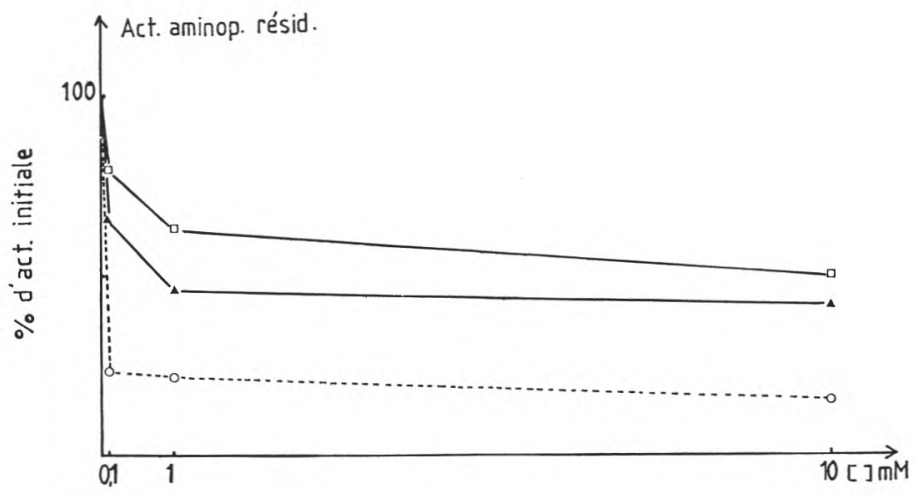

fig. 16

Inactivation de l'extrait enzymatique brut $36_{50}$ par l'E.D.T.A. $\mathbf{A}$, le P.M.S.F. O, la L-cystéine-HCl $\square$ (substrat : L-leucine-para-nitroanilide).

\section{DISCUSSION ET CONCLUSION}

Les informations sur la production et les propriétés du système enzymatique protéolytique de l'espèce $A$. hydrophila sont peu nombreuses (Gross et Coles, 1969).

La majorité des travaux concernent les espèces $A$. proteolytica (Prescott et al., 1960-1974, Foster et Hanna, 1974), A liquefaciens et A. salmonicida (Dahle et al., 1969-1971, Shieh et Mac Lean, 1975). 
Les résultats observés, intéressant la caractérisation du système enzymatique protéolytique d'A. hydrophila LP 50 nous suggèrent les remarques suivantes :

Les températures et $\mathrm{pH}$ optimums d'hydrolyse des protéines varient en fonction de la nature du substrat. Sur caséine, l'activité protéolytique (endopeptidasique) présente un maximum à $50^{\circ} \mathrm{C}$ pour un $\mathrm{pH}$ de $8-8,2(-9)$. En revanche, lorsque l'hémoglobine dénaturée et l'azocaséine sont utilisées comme substrats, la température optima est de $40^{\circ} \mathrm{C}$ pour un $\mathrm{pH}$ optimum voisin de 7,5-8 (7-8,2). Ce décalage dans les optimums de température et de $\mathrm{pH}$ suivant les substrats a déjà été observé par différents auteurs, parmi lesquels Gripon et Bergère [7] étudiant le système protéolytique de Penicillium roqueforti, Saheb [14] qui purifie et caractérise une protéase extracellulaire à partir de Staphylococcus aureus : l'activité protéolytique, dans les deux cas, est déterminée sur caséine et hémoglobine dénaturée.

Très peu de données se rapportent à la température optima d'activité enzymatique. Shieh et Mac Lean [15] mettent en évidence, pour la protéase extracellulaire purifiée à partir d'A. salmonicida, une activité protéolytique sur caséine maxima à $60^{\circ} \mathrm{C}$.

Les $\mathrm{pH}$ optimums mentionnés ici sont comparables à ceux rapportés dans les études de Gross et Coles $(\mathrm{pH} 8$-9) [9], Dahle $(\mathrm{pH} \mathrm{7,9}$ et 9) [3], Shieh et Mac Lean ( $\mathrm{pH} 8$-11) [15] concernant l'action de protéases purifiées sur de la caséine.

Nous n'avons pas observé de différences significatives dans les caractères de l'activité endopeptidasique entre les extraits enzymatiques bruts $36_{50}$ et $54_{60}$, il semble donc qu'aux temps de $36 \mathrm{~h}$ et de $54 \mathrm{~h}$, pour les taux de précipitation de 50 et 60 p. 100 de saturation en sulfate d'ammonium, le système protéolytique soit le même.

A. hydrophila LP 50 possède, en outre, sur L-leucine-para-nitroanilide, une activité aminopeptidasique optima pour une température de $55^{\circ} \mathrm{C}$ et un $\mathrm{pH}$ de $7,5(-8)$. Cette activité, par son $\mathrm{pH}$, se rapproche de l'aminopeptidase de Bacillus stearothermophilus [13] et de l'aminopeptidase de Penicillium roqueforti [8], possédant respectivement vis-à-vis du substrat L.N.A. un $\mathrm{pH}$ optimum de 7,5 et 8 .

L'action des inhibiteurs de l'activité enzymatique révèle que le système enzymatique protéolytique non purifié d'A. hydrophila LP 50 présente les caractères des métalloenzymes, par suite de l'inactivation obtenue avec l'E.D.T.A., la présence de métal n'a cependant pas été recherchée. Ce système enzymatique est également inhibé par le P.M.S.F. et la L-cystéine-HCl, laissant supposer que des résidus séryl et résidus d'acide aminé soufré présenteraient un rôle dans ses activités.

Ces résultats concordent avec les travaux de Gross et Coles [9] qui constatent la perte complète d'activité d'une protéinase produite 
par A. hydrophila, après traitement par l'E.D.T.A. (1 mM). Prescott [12], Griffin [5, 6], Wilkes et al. [17] isolent, à partir d'A. proteolytica, des métalloenzymes, telles que endopeptidase et aminopeptidase, contenant du zinc, inhibées par la cystéine et non affectées par le D.F.P. Selon Dahle [3], l'E.D.T.A. réduit de moitié l'activité de la protéinase A d'A. liquefaciens. Shieh et Mac Lean [15] purifient, dans le cas d'A. salmonicida, une protéase extracellulaire inhibée par le P.M.S.F.

La mise en évidence d'un tel système enzymatique, à partir d'une souche d'Aeromonas provenant de laits pasteurisés, pose le problème plus général des Aeromonas en bactériologie alimentaire. L'important pouvoir protéolytique observé nous suggère que les enzymes protéolytiques des Aeromonas peuvent intervenir au niveau de l'altération des laits, produits laitiers et produits carnés, dont le $\mathrm{pH}$ est voisin de la neutralité ou de l'alcalinité.

Peut-être pourrions-nous aller jusqu'à dire que ces bactéries, fréquemment rencontrées dans les eaux, risquent de jouer un certain rôle dans la dégradation des protéines des produits relevant de la pisciculture !

Des essais de fractionnement et de purification du système enzymatique d'Aeromonas hydrophila LP 50 apparaissent nécessaires pour identifier les protéases responsables des activités endopeptidasique et aminopeptidasique, mises en évidence dans notre travail.

\section{R és u m é}

Les caractères du système enzymatique protéolytique d'Aeromonas hydrophila LP 50 ont été déterminés sur différents extraits enzymatiques bruts obtenus à partir des filtrats acellulaires et choisis suivant leurs activités protéolytiques préalablement étudiées en fonction de la croissance de la souche.

Sur caséine, l'activité endopeptidasique présente un maximum à $50^{\circ} \mathrm{C}$ pour un $\mathrm{pH}$ de $8-8,2$.

Lorsque l'hémoglobine dénaturée et l'azocaséine sont utilisées comme substrats, la température optima est de $40^{\circ} \mathrm{C}$ pour un $\mathrm{pH}$ optimum voisin de 7,5-8.

L'activité aminopeptidasique, sur L-leucine-para-nitroanilide, est optima pour une température de $55^{\circ} \mathrm{C}$ et un $\mathrm{pH}$ de 7,5 .

L'activité aminopeptidasique semble moins thermolabile que l'activité endopeptidasique.

Ce système enzymatique protéolytique exocellulaire, actif à $\mathrm{pH}$ neutre ou légèrement alcalin, est inhibé par l'E.D.T.A., le P.M.S.F. et la L-cystéine-HCl. 


\section{S u m m a r y}

\section{CHARACTERISTICS OF THE PROTEOLYTIC ENZYMATIC SYSTEM OF AEROMONAS HYDROPHILA LP 50}

The characteristics of the proteolytic enzymatic system of Aeromonas hydrophila LP 50 have been determined on different crude enzymatic extracts obtained from acellular filtrates and selected following their proteolytic activities previously studied according to the growth of the strain.

The endopeptidase activity, on casein, presents a maximum at $50^{\circ} \mathrm{C}$ for a $\mathrm{pH}$ of $8-8,2$. When the denatured hemoglobin and azocasein are used as substrates, the optimum temperature is $40^{\circ} \mathrm{C}$ for an optimum $\mathrm{pH}$ about 7,5-8.

The aminopeptidase activity, on L-leucin-para-nitroanilid, is optimum for a temperature of $55^{\circ} \mathrm{C}$ and a $\mathrm{pH}$ of 7,5.

The aminopeptidase activity seems less thermolabile than the endopeptidase activity.

This exocellular proteolytic enzymatic system, active at neutral $\mathrm{pH}$ or slightly alkaline, is inhibited by the E.D.T.A., the P.M.S.F. and the L-cystein-HCl.

Reçu pour publication en février 1980.

\section{Bibliographie}

[1] ANSoN (M. L.) (1938). - Estimation of pepsin, trypsin, papain and cathepsin with hemoglobin. J. Gen. Physiol., 22, 79-89.

[2] Charney (J.) and Tomarelly (R. M.) (1947), - A colorimetric method for the determination of the proteolytic activity of duodenal juice. J. Biol. Chem., $171,501-505$.

[3] Dahle (H. K.) (1971). - The purification and some properties of two Aeromonas proteinases. Acta path. microbiol. Scand, section B, 79, 726-738.

[4] Foster (B. G.) and Hanna (M. O.) (1974). - Toxic properties of Aeromonas proteolytica. Can. J. Microb., 20, 1403-1409.

[5] GRIFFIN (T. B.) (1966). - Production, isolation and physical characterization of Aeromonas proteolytica endopeptidase. Dissert. Biochim. Nutrit., Texas $\mathrm{A}$ and $\mathrm{M}$, University, $82 \mathrm{p}$.

[6] Griffin (T. B.) and Prescott (J. M.) (1970). - Some physical characteristics of a proteinase from Aeromonas proteolytica. J. Biol. Chem., 245, 1348-1356.

[7] Gripon (J. C.) et Bergère (J. C.) (1972). - Le système protéolytique de Penicillium roqueforti. I. Conditions de production et nature du système protéolytique. Le Lait, 518, 497-514.

[8] GRIPON (J.C.) (1977). - The proteolytic system of Penicillium roqueforti. V. Purification and properties of an alkaline aminopeptidase. Biochimie, $59,679-686$.

[9] GRoss (R.) and Coles (N. W.) (1969). - A proteinase produced by Aeromonas hydrophila. Aust. J. Sci., 31, 9, 330-331. 
[10] Lowry (O. H.), Rosebrough (N. J.), FarR (A. L.) and Randall (R. J.) (1951). Protein measurements with the folin phenol reagent. J. Biol. Chem., 193, 265-275.

[11] Methods of enzymatic analysis. Edited by Bermayer. Academic Press, New York and London, 1963, p. 830.

[12] Prescott (J. M.) and Wilkes (S. H.) (1966). - Aeromonas aminopeptidase: purification and some general properties. Arch. Biochem. Biophys., 117, 2, 328-336.

[13] Roncari (G.) and Zuber (H.) (1969). - Thermophilic aminopeptidases from Bacillus stearothermophilus. I. Isolation, specificity and general properties of the thermostable aminopeptidase. Int. J. Protein Res., 1, 45-61.

[14] SAHEB (S. A.) (1976). - Purification et caractérisation d'une protéase extracellulaire de Staphylococcus aureus inhibée par l'E.D.T.A. Biochimie, 58, 793-804.

[15] Shieh (H. S.) and Mac Lean (J. R.) (1975). - Purification and properties of an extracellular protease of Aeromonas salmonicida, the causative agent of furunculosis. Int. J. Biochem., 6, 653-656.

[16] VeIllet-Poncet (L.) (1973). — La flore bactérienne indologène aéro-anaérobie des laits pasteurisés conditionnés. Cas particulier d'Aeromonas. Thèse Doctorat Sciences Naturelles, Univ. Nancy, U.E.R., Alimentation et Nutrition, $\mathrm{n}^{\circ}$ C.N.R.S. AO. 9322. Le Lait, 54, 537, 409-414 ; 538, 537-552 ; 539-540, 675-684.

[17] Wilkes (S. H.), Mukherjee (B. B.), Wagner (F. W.) and Prescott (J. M.) (1969). - Partial purification and some enzymatic properties of a proteinase from Aeromonas proteolytica. Proc. Soc. Exp. Biol. Med., 131, 2, 382-387. 